\title{
A 19-Gene expression signature as a predictor of survival in colorectal cancer
}

Nurul Ainin Abdul Aziz', Norfilza M. Mokhtar ${ }^{2 *}$, Roslan Harun ${ }^{1}$, Md Manir Hossain Mollah', Isa Mohamed Rose ${ }^{3}$, Ismail Sagap ${ }^{4}$, Azmi Mohd Tamil ${ }^{5}$, Wan Zurinah Wan Ngah $^{1}$ and Rahman Jamal ${ }^{*}$

\begin{abstract}
Background: Histopathological assessment has a low potential to predict clinical outcome in patients with the same stage of colorectal cancer. More specific and sensitive biomarkers to determine patients' survival are needed. We aimed to determine gene expression signatures as reliable prognostic marker that could predict survival of colorectal cancer patients with Dukes' B and C.
\end{abstract}

Methods: We examined microarray gene expression profiles of 78 archived tissues of patients with Dukes' B and $C$ using the Illumina DASL assay. The gene expression data were analyzed using the GeneSpring software and $R$ programming.

Results: The outliers were detected and replaced with randomly chosen genes from the $90 \%$ confidence interval of the robust mean for each group. We performed three statistical methods (SAM, LIMMA and t-test) to identify significant genes. There were 19 significant common genes identified from microarray data that have been permutated 100 times namely NOTCH2, ITPRIP, FRMD6, GFRA4, OSBPL9, CPXCR1, SORCS2, PDC, C12orf66, SLC38A9, OR10H5, TRIP13, MRPL52, DUSP21, BRCA1, ELTD1, SPG7, LASS6 and DUOX2. This 19-gene signature was able to significantly predict the survival of patients with colorectal cancer compared to the conventional Dukes' classification in both training and test sets $(p<0.05)$. The performance of this signature was further validated as a significant independent predictor of survival using patient cohorts from Australia $(n=185)$, USA $(n=114)$, Denmark $(n=37)$ and Norway $(n=95)(p<0.05)$. Validation using quantitative PCR confirmed similar expression pattern for the six selected genes.

Conclusion: Profiling of these 19 genes may provide a more accurate method to predict survival of patients with colorectal cancer and assist in identifying patients who require more intensive treatment.

Keywords: Colorectal cancer, Microarray analysis, Survivalm, Real-time PCR

Abbreviations: cDNA, Complementary deoxyribonucleic acid; CRC, Colorectal cancer; DASL, CDNA-mediated annealing, selection, extension and ligation; DE, Differentially expressed; FFPE, Formalin-fixed paraffin embedded; GEO, Gene Expression Omnibus; LIMMA, Linear Model for Microarray Data; PCR, Polymerase chain reaction; rCl, Robust confidence interval; RMA, Robust Multichip Average; RNA, Ribonucleic acid; RT-PCR, Real-time polymerase chain reaction; SAM, Significant analysis of microarray; UKM, Universiti Kebangsaan Malaysia; USA, United States of America

\footnotetext{
* Correspondence: norfilza@ppukm.ukm.edu.my; rahmanj@ppukm.ukm.edu.my

${ }^{2}$ Department of Physiology, Faculty of Medicine, Universiti Kebangsaan

Malaysia, Jalan Yaacob Latif, Bandar Tun Razak, Cheras, 56000 Kuala Lumpur,

Malaysia

${ }^{1}$ UKM Medical Molecular Biology Institute, Universiti Kebangsaan Malaysia

(UKM), Cheras, Kuala Lumpur, Malaysia

Full list of author information is available at the end of the article
} 


\section{Background}

Colorectal cancer is one of the major causes of cancer mortality in both sexes worldwide. The reported number of CRC patients has increased to 1.4 million and associated with 694 000 deaths globally in 2012 [1]. CRC is staged according to the extent whether it has spread through the wall of colon and rectum or to other parts of the body [2]. The prognosis is influenced by the stage of CRC at the time of diagnosis [3]. Based on the National Cancer Institute's Physician Data Query system, the 5-year survival rate for Dukes' A patients was 80 to 95 \%, Dukes' B 55 to $80 \%$, Dukes' C 33 to $55 \%$ and Dukes' D less than $15 \%$ [4]. These data showed the correlation between survival and staging where the higher stage of CRC patients is associated with a lower survival rate. However, a previous study reported that the survival rate of Dukes' B patients with high risk pathological factors or low nodes involvement was lower than Dukes' $\mathrm{C}$ patients who had one positive node [3]. Thus, the current staging method needs to be improved to provide a more accurate prognostication for CRC patients. The common practice in managing Dukes' B patients is a combination of surgery, chemotherapy and/or radiation therapy [5]. Whether this should be applied for all cases is still debatable [3]. The adjuvant chemotherapy may benefit the Dukes' B patients with high risk features but this is still not routinely recommended. This is due to less benefit obtained from the adjuvant chemotherapy as 10-20\% of patients will develop recurrence [6]. For Dukes' $\mathrm{C}$ patients, the adjuvant chemotherapy became a standard treatment after showing a $40 \%$ reduction of recurrence rate [7]. Another study in 2004 has demonstrated that the overall 5-year survival rate was poor in patients with Stage IIb compared to those with stage IIIa [4]. However, this result may be due to the misclassification of staging which leads to poor survival in untreated patients with micrometastasis [4]. Clearly, there are pitfalls in using the current staging system for prognostication purposes.

Nowadays, the development of high throughput technologies such as RNA sequencing $[8,9]$ and microarray $[10,11]$ become popular to generate gene expression profiling in understanding of cancer. Microarray technology is still valuable and promising technology for many years as it is more affordable compared to the RNA sequencing. Eschrich et al. (2005) used cancer tissues from patients with Dukes' B, C and D, who have been follow-up for at least 36 months. They found a 43-gene signature that categorize patients into good and poor survival with $93 \%$ sensitivity and $84 \%$ specificity [12]. But, a large scale validation could not be performed due to the limitation to make decision for adjuvant treatments [11, 13]. Several studies that analyzed patients with Stage II and III CRC have developed molecular classifiers that could stratify patients into high and lowrisk groups [14-16]. However, these studies are still in the research phase were not been translated into clinical practice [17]. Furthermore, some studies have used a small number of samples and lack of validation in independent samples to enhance the power of the gene signatures [18]. Our aim for this study was to determine gene expression signatures that could predict survival of CRC patients with Dukes' B and C CRC, hoping that a set of gene signatures will be able to classify patients into those with good or poor survival as well as to more accurately targeted therapy.

\section{Methods}

\section{Clinical samples}

This is a retrospective study using 78 formalin-fixed paraffin embedded (FFPE) tissues of patients with Dukes' B $(n=37)$ and Dukes' C $(n=41)$ CRC patients diagnosed between January 2002 to December 2007 at the Universiti Kebangsaan Malaysia Medical Centre. These samples comprised of patients who survived less than five years (denoted as the poor survival group) and patients who survived more than five years (good survival group). The samples were anonymised throughout this study. Inclusion criteria included the absence of preoperative chemotherapy or radiotherapy. Information about age, gender, race, histology, family history, organ sites and clinical outcomes were recorded. For each patient, their medical records and follow-up data were carefully reviewed to confirm their clinical outcomes and the cause of death if the patients were deceased. The survival of patients was calculated from December 2012 minus the date of the first surgery for those still alive while for those who did not survive, it included the date of death minus the date of the first surgery.

\section{RNA extraction}

Tissue sections of 4-7 $\mu \mathrm{m}$ in thickness were prepared (>80 \% representative), stained with hematoxylin and eosin (H\&E) and evaluated by the pathologist in charge. RNA was deparaffinized and extracted using High Pure RNA Paraffin Kit (Roche Applied Science, Mannheim, Germany). Proteinase $\mathrm{K}$ was added and homogenization was performed for $16 \mathrm{~h}$. All steps followed the manufacturer's protocol. Samples were then stored at $-80{ }^{\circ} \mathrm{C}$ until they were used. Quantity and purity of the total RNA was determined by the NanoDrop ND-1000 spectrophotometer (Thermo Fisher Scientific, Waltham, MA). Samples with purity between 1.8 to 2.0 (A260/ A280) were selected. Quality assessment of total RNA was done using the Bioanalyzer 2100 RNA 6000 Nano kit (Agilent Technologies, Inc., CA, USA) and samples with RNA Integrity Number (RIN) of more than two were selected for the quantitative PCR. 
cDNA mediated annealing, selection, extension and ligation (DASL) assay

Quantitative PCR analysis was performed as the prequalifying step prior to cDNA synthesis using the Corbett Rotor-Gene 6000 thermal cycler (Corbett Life Science, Sydney, Australia). Forward and reverse primers for the housekeeping gene RPL13A were obtained from AITbiotech Singapore. PCR amplification with $C_{T}$ value of 29 cycles or less was used in DASL assay (Illumina, San Diego, CA, USA). The assay was conducted according to the manufacturer's protocol. Raw data files (.idat files) were analyzed using the GenomeStudio software (Illumina, San Diego, CA, USA) to check the data quality control for assessing results of gene expression microarray experiment.

\section{Microarray analysis}

Sample Probe Profile from the GenomeStudio was imported to the third party software called Genespring GX 12.0.2 (Agilent Technologies, Inc., CA, USA). Seventy-eight samples were exploitable with 20793 entities. The data were normalized using quantile algorithm and log-transformed. Baseline transformation of the normalized signal was performed to the median of all samples. Samples were assigned into their survival groups. Hierarchical clustering was performed using Pearson's correlation coefficient and Ward's criterion.

\section{Outlier diagnosis for microarray analysis}

It is well known that microarray gene-expression data are often contaminated by outliers due to many steps involved in the experimental process from hybridization to image analysis $[19,20]$. Most of the popular algorithms for microarray gene-expression data analysis are very much sensitive to outliers [19]. So geneexpression data analysis by these algorithms may produce misleading results in the presence of contaminated observations.

We identified the contaminated observations for each gene using $\beta$-weight function $[21,22]$ and replaced them with the values belonging to the $90 \%$ robust confidence interval $(\mathrm{rCI})$ of the respective group mean. The $90 \% \mathrm{rCI}$ for the $j$-th group mean $\left(\mu_{i}^{(j)}\right)$ of $i$-th gene is defined by

$$
\left[\left(\hat{\mu}_{i, \beta}^{(j)}-1.644 \times \hat{\sigma}_{i, \beta}^{(j)} / \sqrt{n_{j}}\right),\left(\hat{\mu}_{i, \beta}^{(j)}+1.644 \times \hat{\sigma}_{i, \beta}^{(j)} / \sqrt{n_{j}}\right)\right]
$$

where $\hat{\mu}_{i, \beta}^{(j)}$ and $\hat{\sigma}_{i, \beta}^{(j)}$ are the minimum $\beta$-divergence estimators of mean $\left(\mu_{i}^{(j)}\right)$ and variance $\left(\sigma_{i}^{2(j)}\right)$ obtained iteratively as follows

$$
\mu_{i, t+1}^{(j)}=\frac{\sum_{k=1}^{n_{j}} \psi_{\beta}\left(x_{i k}^{(j)} \mid \theta_{i, t}^{(j)}\right) x_{i k}^{(j)}}{\sum_{k=1}^{n_{j}} \psi_{\beta}\left(x_{i k}^{(j)} \mid \theta_{i, t}^{(j)}\right)}
$$

and

$$
\sigma_{i, t+1}^{2(j)}=\frac{\sum_{k=1}^{n_{j}} \psi_{\beta}\left(x_{i k}^{(j)} \mid \theta_{i, t}^{(j)}\right)\left(x_{i k}^{(j)}-\mu_{i, t}^{(j)}\right)^{2}}{(\beta+1)^{-1} \sum_{k=1}^{n_{j}} \psi_{\beta}\left(x_{i k}^{(j)} \mid \theta_{i, t}^{(j)}\right)}
$$

where $\theta_{i}^{(j)}=\left(\mu_{i}^{(j)}, \sigma_{i}^{2(j)}\right), x_{i k}^{(j)}$ is the $k$-th expression in group- $j$ of gene- $i, i=1,2, \ldots ., N=20793 ; j=1,2 ; n_{1}+n_{2}=78$, and which is known

$$
\psi_{\beta}\left(x_{i k}^{(j)} \mid \theta_{i}^{(j)}\right)=\exp \left\{-\frac{\beta}{2}\left(\frac{x_{i k}^{(j)}-\mu_{i}^{(j)}}{\sigma_{i}^{(j)}}\right)^{2}\right\}
$$

as $\beta$-weight function that we used for outlier detection as mentioned earlier. This weight function produces weights between 0 and 1 for any observation detected. It produces smaller weights only for contaminated observations. So, in this study we consider an observation $\left(x_{i k}^{(j)}\right)$ as a contaminated observation when

$$
\psi_{\beta}\left(x_{i k}^{(j)} \mid \hat{\theta}_{i, \beta}^{(j)}\right)<0.2
$$

and replaced it with a value belonging to the $90 \%$ rCI of mean $\left(\mu_{i}^{(j)}\right)$ as the defined eq. (1). The $\beta$ estimators as defined in eqs. 2 and 3 are highly robust against outliers [21, 22].

\section{Detection of differentially expressed (DE) gene}

We permutated 100 times from one data the microarray data obtained from78 patients. All patients were divided into two subsets of equal numbers i.e., training and test sets. We used the bootstrapped data with three statistical methods (SAM, LIMMA and $t$-test) to each training and test set to detect significantly DE genes between good and poor survival group.

\section{Survival analysis}

Cox proportional hazards model and Elastic net estimation

To estimate the relationship between the survival time and the gene expression levels, we used $n$ as a sample of $\mathrm{n}$ size and $X_{1}, \ldots, X_{p}$ of $p$ genes to denote the gene expression level. The survival data for the $i$ th patient denoted by $\left(T_{\dot{v}} \delta_{\dot{v}} x_{i 1}, x_{i 2} \ldots x_{i p}\right)$, where $i=1,2, \ldots, n, T i$ is the survival time of $i$ patient, $\delta_{i}$ is censoring 
indicator ( 0 if alive, 1 death) and $x_{i}=\left\{x_{i 1}, x_{i 2} \ldots, x_{i p}\right\}$ is the vector of the gene expression level of $p$ genes (covariates). We also used the Cox regression model for the hazard of CRC death at time $t$ which is defined by

$$
\begin{aligned}
\lambda(t) & =\lambda o(t) \exp \left(\beta_{1} X_{1}+\beta_{2} X_{2}+\cdots+\beta_{p} X_{p}\right) \\
& =\lambda_{0}(t) \exp \left\{\beta^{T} X\right\},
\end{aligned}
$$

where $\lambda_{0}(t)$ is an unspecified baseline hazard function, $\beta=\left\{\beta_{1}, \beta_{2}, \cdots, \beta_{\mathrm{p}}\right\}$ is the vector of regression coefficients and $X=\left\{X_{1}, \ldots, X_{p}\right\}$ is the vector of gene expression levels with the corresponding sample values of $x i=\left\{x_{i 1}, \ldots, x_{i p}\right\}$ for the $i$ th sample. The the risk score of patient was calculated from the function:

$$
\text { Risk Score }=f(X)=\beta^{T} X
$$

Based on the available sample data, the Cox's partial likelihood can be written as

$$
L(\beta)=\prod_{r \in D} \frac{\exp \left(\beta^{T} x_{r}\right)}{\sum_{j \in R_{r}} \exp \left(\beta^{T} x_{j}\right)}
$$

where $D$ is the set of indices of the events (e.g., deaths) and $R_{\mathrm{r}}$ denotes the set of indices of the individuals at risk at time $t_{\mathrm{r}}-0$. The Elastic Net [23] uses a mixture of the $\mathrm{L}_{1}$ (lasso) and $\mathrm{L}_{2}$ (ridge regression) penalties. In the Elastic Net, the usual partial log-likelihood is penalized by the $\mathrm{L}_{1}$ and $\mathrm{L}_{2}$ norms of the regression coefficients with weights $\lambda_{1}$ and $\lambda_{2}$, respectively, i.e.,:

$$
l(\beta)_{\text {penalized }}=l(\beta)-\lambda_{1} \sum_{i-1}^{p}\left|\beta_{i}\right|-\lambda_{2} \sum_{i-1}^{p}\left(\beta_{i}\right)^{2}
$$

where $\lambda_{1}$ and $\lambda_{2}$ are tuned by maximizing $l(\beta)$, and $l(\beta)$ is the cross-validated partial log-likelihood (CVL). LASSO and Ridge regression are described by Eq. (2) with $\lambda_{1}$ or $\lambda_{2}$ non-zero, respectively. The $\lambda_{1}+\lambda_{2}$ Elastic Net involves 2D optimization of the penalties. The penalty parameters were tuned 50 times using different folding of the data for calculating CVL, and the penalty parameters with maximum CVL were selected by pensim $\mathrm{R}$ package, available at http://cran.r-project.org/web/packages/pensim/index.html.

We performed the Elastic Net [23] using the opt2D function of the "pensim" $\mathrm{R}$ package to predict the survival of CRC patients from microarray data. Using a 10 -fold cross-validation, with 50 starts parallelized to 8 processors using the opt $2 D$ function, we obtained regression coefficients $(\beta)$ with the optimal penalty parameter for the penalized Cox model, and calculated the risk score for each patient using eq. (5) where $\beta_{i}$ is the estimated regression coefficient of each gene in the training data set and $X_{i}$ is the Z-transformed expression value of each gene. The estimated regression coefficient of each survival related gene given by Elastic Net in eq. (6) in the training data set was also applied to calculate a risk score for each patient in test data set. The linear risk score with greatest crossvalidated partial log-likelihood was selected for validation in the test set. We classified all patients into the 2 groups high and low risk groups using the cut-off value (median risk score) in the training set. Patients were assigned to the "high-risk" group if their risk score was more than or equal to cut-off value of risk score, whereas those with less than the cutoff values were assigned as "low-risk". The patients in high-risk group are expected to have a poor outcome. The statistical significance of the predictions was then assessed by the likelihood ratio test on the Cox proportional hazards model. The probe sets were scaled to $\mathrm{z}$-scores per feature for all datasets. An individual patient (test patient) can be checked to predict whether the patient should receive further treatment or no treatment by the fitted risk score (eq. 5), where $X=\left\{X_{1}, \ldots, X_{p}\right\}$ takes the expression values of selected $p=19$ genes from the test patient in the real life daily practice.

The values of specificity and sensitivity of the 19-genes was calculated based on the analysis of gene expression from this study as compared to the selected genes from other publications $[14,15]$.

\section{Independent external validation}

We compared our microarray data with the published datasets obtained from Stage II and III CRC patients from four separate international studies (Australia, USA, Denmark and Norway) [11, 14, 15, 24]. The datasets were accessed online from Gene Expression Omnibus (GEO) (GSE14333, GSE17536/GSE17537, GSE31595 and GSE30378). The platform used was Affymetrix HG-U133 Plus2.0. The raw fluorescence intensity data within CEL files were pre-processed with Robust Multichip Average (RMA) algorithm [8], as implemented with $\mathrm{R}$ packages from Bioconductor (http://www.bioconductor.org), and the data were log-transformed. Clinical information of the publicly available microarray data sets was obtained from the published articles and websites. In addition, the data were normalized per gene in each data set by transforming the expression of each gene to obtain a mean of 0 and SD of 1 (Z-transformation) for this study.

\section{Validation using quantitative PCR (qPCR)}

Six genes (FRMD6, SLC38A9, TRIP13, MRPL52, ELTD1 and ITPRIP) were randomly selected for the validation of the microarray data. Results were normalized with RPL13A gene. The extracted total RNA was converted to cDNA using Verso cDNA Synthesis kit (Thermo Scientific, UK). For qPCR, $25 \mu \mathrm{l}$ reactions were set up using $12.5 \mu \mathrm{l}$ of $2 \mathrm{X}$ Solaris qPCR Master Mix, $1.25 \mu \mathrm{l}$ of Solaris Primer/Probe Set (20X), $1 \mu \mathrm{l}$ of cDNA template and water to make up to total volume $25 \mu$ l. The qPCR reactions were performed using the Rotor-Gene 6000 
thermal cycler (Corbett Life Science). Cycling program involved one cycle of enzyme activation at $95{ }^{\circ} \mathrm{C}$ for $15 \mathrm{~min}, 40$ cycles consist of denaturation at $95{ }^{\circ} \mathrm{C}$ for $15 \mathrm{~s}$ and annealing/extension at $60{ }^{\circ} \mathrm{C}$ for $60 \mathrm{~s}$.

\section{Results}

\section{Clinical and pathological features}

Clinical and pathological features of 78 patients were separated into poor and good survival groups of patients who survived less than five years and more than five years respectively. In this study, the 5 year survival rate among patients of Dukes' B was $59.5 \%$ while Dukes' C was $36.5 \%$. It was in concordance to the United State data [4]. The differences in clinical parameters between Dukes' B and C patients were not statistically significant (Fisher's exact test $p=0.173$ ) (Table 1). Kaplan Meier curves were constructed based on Dukes' staging and the survival time showed no statistically significant difference ( $\log$ rank $p=0.242$, data not shown). Fig. 1 showed the H\&E staining results of patient Dukes' B and C.

\section{Identification of DE genes between good and poor survival groups}

Based on the eqs. (4 \& 4.1), we identified $7.7 \%$ of 20793 probes as contaminated probes (Additional file 1). Then,

Table 1 Clinical and pathological features

\begin{tabular}{|c|c|c|c|c|}
\hline & & \multirow{2}{*}{$\begin{array}{l}\text { Good survival } \\
n=39 \\
\text { No }(\%)\end{array}$} & \multicolumn{2}{|c|}{$\begin{array}{l}\text { Poor survival } \\
n=39\end{array}$} \\
\hline & & & No (\%) & $p$ value \\
\hline \multirow[t]{2}{*}{ Dukes' } & B & $22(56.41)$ & $15(38.46)$ & $0.173 * *$ \\
\hline & C & $17(43.59)$ & $24(61.54)$ & \\
\hline \multirow[t]{2}{*}{ Gender } & Male & $20(51.28)$ & $20(51.28)$ & $1.000 * *$ \\
\hline & Female & $19(48.72)$ & $19(48.72)$ & \\
\hline \multirow[t]{2}{*}{ Age (year) } & $\leq 50$ & $4(10.26)$ & $5(12.82)$ & $0.235^{* *}$ \\
\hline & $>50$ & 35 (89.74) & $34(87.18)$ & \\
\hline \multirow[t]{3}{*}{ Race } & Chinese & $29(74.36)$ & $24(61.54)$ & $0.226 *$ \\
\hline & Malay & $9(23.08)$ & $15(38.46)$ & \\
\hline & Indian & $1(2.56)$ & 0 & \\
\hline \multirow[t]{5}{*}{ Tumor differentiation } & Well & $26(66.67)$ & $15(38.46)$ & $0.051 *$ \\
\hline & Moderately & $5(12.82)$ & $15(38.46)$ & \\
\hline & Poorly & $1(2.56)$ & $2(5.13)$ & \\
\hline & Mucinous & $2(5.13)$ & $4(10.26)$ & \\
\hline & No record & $5(12.82)$ & $3(7.69)$ & \\
\hline \multirow[t]{2}{*}{ Clinical outcome } & Alive & $34(87.18)$ & 0 & $0.000 * *$ \\
\hline & Dead & $5(12.82)$ & $39(100.00)$ & \\
\hline \multirow[t]{2}{*}{ Organ } & Colon & $25(64.10)$ & $21(53.85)$ & $0.357^{* *}$ \\
\hline & Rectum & $14(35.90)$ & $18(46.15)$ & \\
\hline
\end{tabular}

* $=p$ value was calculated using Pearson Chi-Square

$* *=p$ value was calculated using Fisher's Exact Test

[Relevant location: Page 13] we updated all contaminated expressions for each gene using the reasonable values belonging to the $90 \% \mathrm{rCI}$ of their respective group means as discussed earlier.

Thus, a total of 1500 top-ranked DE genes (using smaller adjusted $p$-values) was selected from each of training and test datasets by each of three statistical tests (See Methods). Overlapping genes obtained by three statistical test were again overlapped between each of the training and test datasets (Additional file 1). Finally we obtained 19 significant DE genes (NOTCH2, ITPRIP, FRMD6, GFRA4, OSBPL9, CPXCR1, SORCS2, PDC, C12orf66, SLC38A9, OR10H5, TRIP13, MRPL52, DUSP21, BRCA1, ELTD1, SPG7, LASS6 and DUOX) for further investigation (Table 2).

Figure 2 shows an example of the hierarchical clustering of microarray results based on $19 \mathrm{DE}$ genes from a pair of training set 1 and test set 1.

\section{Predicting survival of cancer patients from CRC gene expression data}

We performed the Elastic Net [23] to the training dataset and compute the risk scores using eq. (5) based on the model estimates to the training dataset and the test dataset. After calculating the risk score for each patient from the 19-gene expression signature as mentioned before, we divided the training set into high and low risk groups based on the cutoff value $(-0.07)$ of the risk score. The likelihood ratio test was used to compare differences in overall survival between high and low risk groups in the training set 1 (likelihood ratio test, $p<0.05$; HR $=27$, (95 \% CI, 5.165 $140.5)$ ) and test set 1 (likelihood ratio test, $p<0.05$, $\mathrm{HR}=12$, (95 \% CI, 2.861 - 47.21)). Both Kaplan Meier survival plots (Fig. 3a and b) for training and test set 1 showed that this risk classification was significantly associated with the overall survival time. Similar results were observed in the other training and test sets. We also compared other two methods such as LASSO and Ridge regression with Elastic Net regression for prediction accuracy in our data. The prognostic index (risk score of 19 gene signature) was significantly associated with overall survival time in multivariate analysis (Table 3).

This study showed that the sensitivity and specificity of the 19 -genes were $86.84 \%$ and $87.50 \%$ respectively which were acceptably higher than the ColoGuideEx with $72.22 \%$ and $71.43 \%$ respectively for 13-genes signature [14]. Meanwhile, ColoGuidePro [15] has the upmost value of analysis with $94.44 \%$ and $90.48 \%$ for 7 -genes respectively. To identify genes that may predict overall survival, a univariate Cox proportion hazard regression analysis was performed with each of 19 differentially expressed genes in CRC in a cohort of 78 patients (Table 4). Table 4 


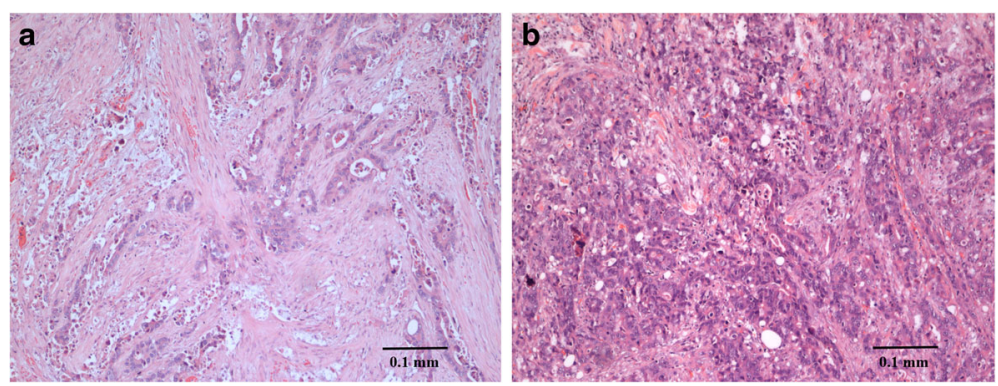

Fig. 1 a Cancerous tissue section of patients Dukes' B well-differentiated adenocarcinoma. Hematoxylin (purple) stains chromatin in the nucleus and eosin (pink orangish) gives color to the protein that resides in the cytoplasm of muscle cells. Tumor cells appear to thicken and be seen spreading muscular propia but did not penetrate serous layer. $\mathbf{b}$. Well differentiated adenocarcinoma Dukes' $\mathrm{C}$ tissue section invaded into muscular propia and involved lymph nodes

shows that most of the genes including validated 5 genes (FRMD6, MRPL52, TRIP13, ELTD1 and SLC38A9) were significantly correlated with the overall survival of the CRC patients. The five validated genes were significantly correlated with the overall survival with hazard ratios of $1.259[p=0.001 ; 95 \%$ confidence interval $(\mathrm{CI}): 1.092$ to 1.452 ], $0.848(p=0.001 ; 95 \% \mathrm{CI}: 0.767$ to 0.939$)$, 0.881 ( $p=0.008$; $95 \%$ CI: 0.802 to 0.968$), 1.155$ ( $p=0.021$; $95 \% \mathrm{CI}): 1.021$ to 1.307$)$ and $0.919(P=0.050$; $95 \% \mathrm{CI}$ : 0.845 to 1 ) respectively.
To determine whether these 19 genes can be independent prognostic markers, multivariate analysis was also performed including other clinical parameters (age, gender and stage) as shown in Table 4 . The results showed that five genes (NOTCH2, GFRA4, OSBPL9, MRPL52 and LASS6) as independent predictors with hazard ratios of $1.56(P=0.009,95 \% \mathrm{CI}: 1.034$ to 1.913), 0.871 ( $p=0.010,95 \%$ CI: 0.547 to 1.005$), 0.818$ $(p=0.047,95 \%$ CI: 0.640 to 1.045$), 0.865(p=0.019$, $95 \% \mathrm{CI}: 0.697$ to 1.075$)$ and $0.788(P=0.035,95 \% \mathrm{CI}$ :

Table 2 Microarray-based changes in gene expression of the 19 genes

\begin{tabular}{|c|c|c|c|c|}
\hline Probe ID & Gene symbol & ${ }^{\mathrm{a}}$ Fold change & Gene name & $\begin{array}{l}\text { Expression in poor survival group } \\
\text { (Up-regulated/Down-regulated) }\end{array}$ \\
\hline 7000692 & MRPL52 & $-4.32(-2.59)$ & mitochondrial ribosomal protein $\mathrm{L} 52$ & Down-regulated \\
\hline 5700373 & TRIP13 & $-3.49(-3.22)$ & thyroid hormone receptor interactor 13 & Down-regulated \\
\hline 2690324 & ITPRIP & $1.36(1.23)$ & inositol 1,4,5-triphosphate receptor interacting protein & Up-regulated \\
\hline 7000184 & SLC38A9 & $-3.89(-3.08)$ & solute carrier family 38, member 9 & Down-regulated \\
\hline 5420070 & FRMD6 & $3.65(2.63)$ & FERM domain containing 6 & Up-regulated \\
\hline 4230739 & SORCS2 & $2.96(3.58)$ & sortilin-related VPS10 domain containing receptor 2 & Up-regulated \\
\hline 6040070 & ELTDI & $3.68(2.59)$ & EGF, latrophilin and seven transmembrane domain containing 1 & Up-regulated \\
\hline 1190176 & NOTCH2 & $3.37(2.62)$ & Notch homolog 2 & Up-regulated \\
\hline 2570196 & CPXCR1 & $-2.62(-2.18)$ & CPX chromosome region, candidate 1 & Down-regulated \\
\hline 840367 & $\mathrm{OR} 10 \mathrm{H} 5$ & $-2.20(-1.93)$ & olfactory receptor, family 10, subfamily $\mathrm{H}$, member 5 & Down-regulated \\
\hline 3450575 & PDC & $-3.06(-1.75)$ & phosducin & Down-regulated \\
\hline 2710564 & DUOX2 & $2.22(3.01)$ & dual oxidase 2 & Up-regulated \\
\hline 4560474 & GFRA4 & $-2.91(-1.63)$ & GDNF family receptor alpha 4 & Down-regulated \\
\hline 5690064 & LASS6 & $-2.69(-2.45)$ & LAG1 homolog, ceramide synthase 6 & Down-regulated \\
\hline 3780725 & OSBPL9 & $-2.04(-2.45)$ & oxysterol binding protein-like 9 & Down-regulated \\
\hline 5090025 & C12orf66 & $-1.15(-1.11)$ & chromosome 12 open reading frame 66 & Down-regulated \\
\hline 5870121 & SPG7 & $3.64(4.57)$ & spastic paraplegia 7 (pure and complicated autosomal recessive) & Up-regulated \\
\hline 620398 & DUSP21 & $-3.53(-2.92)$ & dual specificity phosphatase 21 & Down-regulated \\
\hline 540411 & BRCA1 & $-3.88(-3.07)$ & breast cancer 1 , early onset & Down-regulated \\
\hline
\end{tabular}

This table shows the probe ID, gene symbols and expression of the 19 genes in the poor survival group compared to the good survival group. ${ }^{\mathrm{a}}$ Fold change: training set (test set)

[Relevant location: Page 13] 


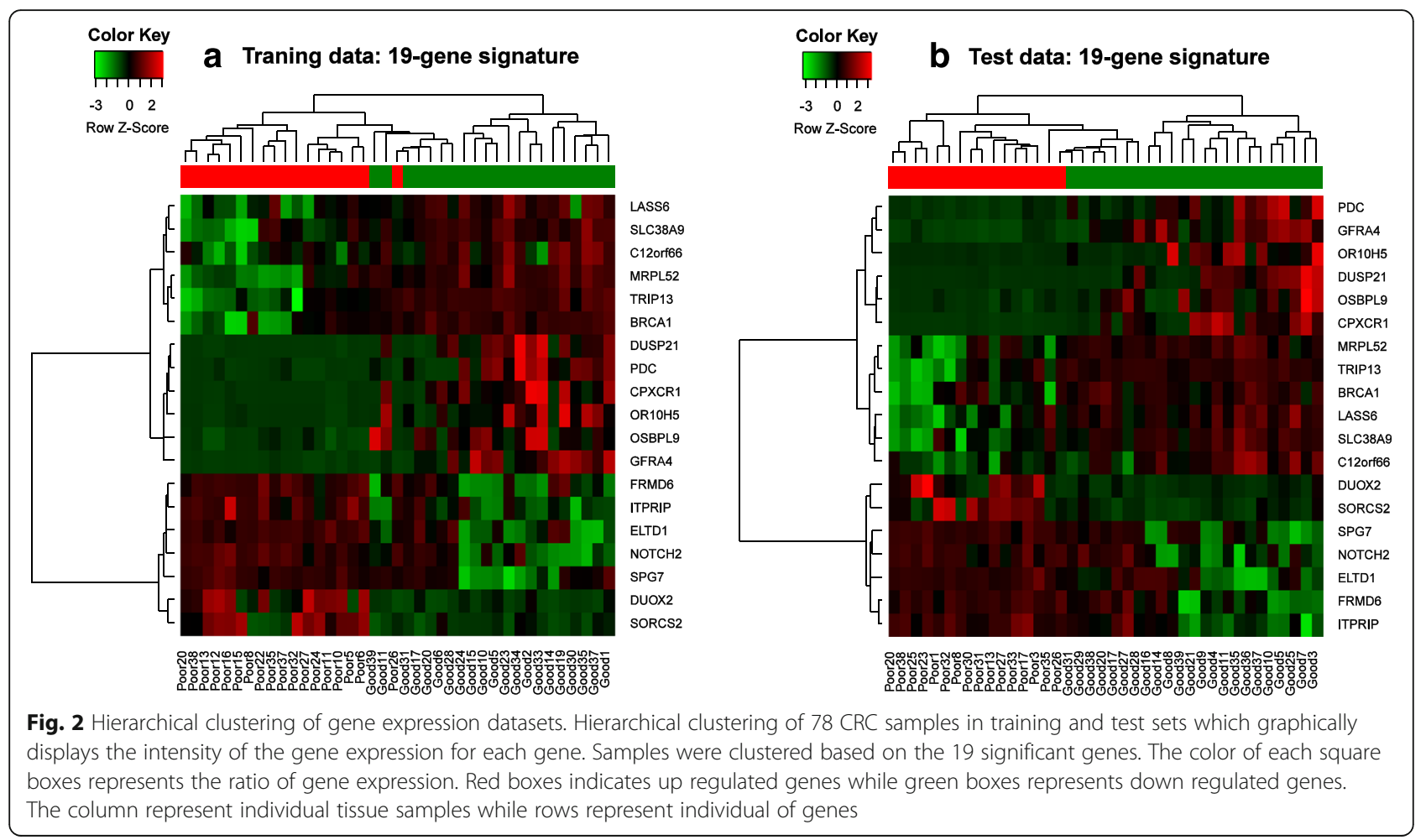

0.631 to 0.984 ) respectively. To improve the prognostic capability, a risk score was calculated based on the expression level of NOTCH2, GFRA4, OSBPL9, MRPL52 and LASS6 and corresponding regression coefficients. A patient's risk score was calculated as the sum of the expression values of these genes. The results confirmed that the patients in the low-risk score group also had a better prognosis than those in the high-risk score group in the test set. This data suggest that the risk score based on these five genes can be used to stratify patients (Fig. 3c).

Survival analysis of the 19-gene signature using the USA, Australia, Denmark and Norway datasets

We assessed the predictive power of the 19 gene signatures on the four cohort datasets from the USA $(n=$ $114)$, Australia $(n=185)$, Denmark $(n=37)$ and Norway $(n=95)$. All the microarray data were obtained using the Affymetrix platform and we confirmed that the risk classification using the 19 genes were replicated in all three datasets. We found 17 out of 19 gene signatures were present in the datasets from the USA, Australia and Norway while 18 out of 19 genes were present in the dataset from Denmark.

The Kaplan Meier survival curves for the high and low risk score groups are shown in Fig. 3d-f. Patients with high risk scores showed significantly poorer overall survival than the patients with low risk scores for the
USA dataset (likelihood ratio test $p$-value $<0.01$; HR $=$ 4.9 (95\% CI, 1.827 - 12.99)), for the Australian dataset, (likelihood ratio test $p$-value $<0.01 ; \quad H R=1.8$ (95\% CI, $1.268-2.533)$ ) and for the dataset from Denmark (likelihood ratio test $p$-value $<0.01 ; H R=8.6$ (95\% CI, 1.842 - 40.05)). Interestingly, we observed that the median risk score for all external validation datasets as well as our training dataset situated between -0.099 and -0.038 . We again compared LASSO and Ridge with Elastic Net regression for prediction patients into high and low risk survival groups in external datasets from different countries. The prognostic index was significantly associated with overall survival time in most of the external datasets in multivariate analysis (Table 3). We observed that the LASSO and Ridge methods fail to obtained prognostic index to measure the association, while the elastic net was significantly associated with overall survival time in the Norway dataset (Table 3).

\section{Validation of the microarray data}

Validation using qPCR demonstrated similar trends between poor and good survival groups when compared with the microarray data. All up-regulated genes (FRMD6, ELTD1 and ITPRIP) and down-regulated genes (MRPL52, TRIP13 and SLC38A9) were confirmed by qPCR according to $2^{-\Delta \Delta C}$ method as seen in Fig. 4. 

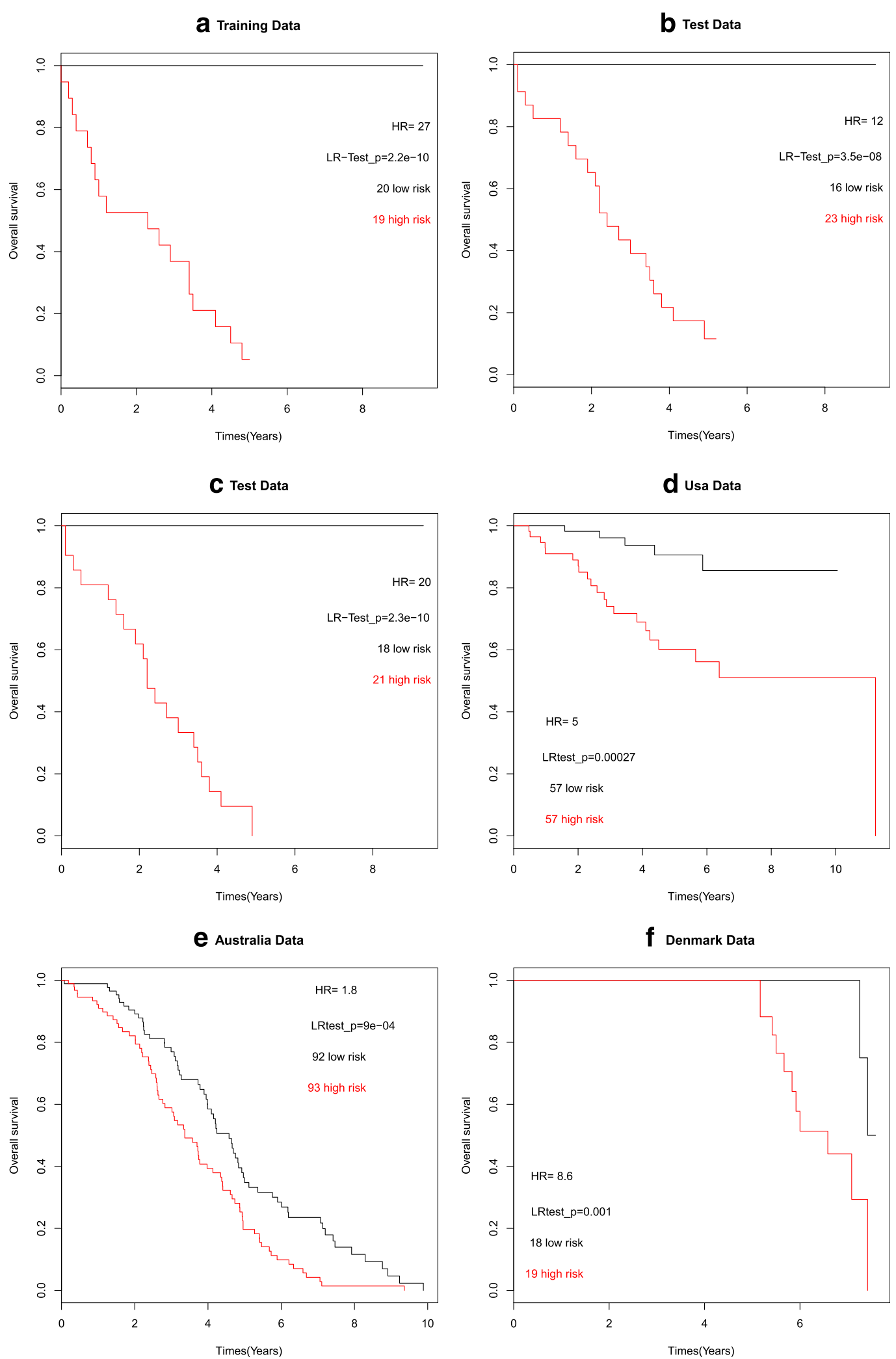

Fig. 3 Survival analysis. Kaplan-Meier survival analysis using six different microarray datasets (Training and test sets (Illumina-based), dataset from Denmark (Affymetrix), dataset from the USA (Affymetrix) and dataset from Australia (Affymetrix)). The 19-gene signature segregates patients into two risk groups (red, high risk; black, low risk). The p values correspond to the likelihood ratio test comparing the survival curves 
Table 3 Univariate and multivariate cox proportional hazard regression analyses

\begin{tabular}{|c|c|c|c|c|}
\hline \multirow[t]{2}{*}{ Genes } & \multicolumn{2}{|l|}{ Univariate } & \multicolumn{2}{|l|}{ Multivariate } \\
\hline & Hazard ratio $(95 \%$ Cl) & $p$-value & Hazard ratio (95 \% Cl) & $p$-value \\
\hline TCH2 & $1.356(1.154-1.592)$ & $0.000 * * *$ & $1.56(1.034-1.913)$ & $0.009 * *$ \\
\hline tim & $1.063(0.919-1.23)$ & 0.408 & 1 ז & 0.340 \\
\hline FRM 6 & $1.259(1.092-1.452)$ & $0.001 * *$ & $1.066(0.850-1.338)$ & 0.575 \\
\hline BA4 & $0.860(0.728-1.016)$ & * & $0.871(0.547-1.005)$ & 0.010 * \\
\hline OSBPL9 & $0.803(0.671-0.962)$ & $0.017 * *$ & $0.818(0.640-1.045)$ & $0.047^{* *}$ \\
\hline CPXCR1 & 0918 & $83^{\prime}$ & $5-1.387)$ & 0.840 \\
\hline ORCS2 & $1.159(1.041-1.290)$ & $0.006^{* *}$ & $0.936(0.752-1.166)$ & 0.558 \\
\hline PDC & 0.812 & * & 1.068 & 0.659 \\
\hline $20 r f 66$ & 0.902( & 0.06 & 1.007 & 0.965 \\
\hline SLC38 & 0.919 & $0.050 *$ & $1.073(0.894-1$ & 0.443 \\
\hline $\mathrm{R} 10 \mathrm{H} 5$ & $0.846(0.704-1.018)$ & $0.075^{*}$ & $1.078(0.826-1.407)$ & 0.579 \\
\hline TRIP13 & $0.881(0.802-0.968)$ & $0.008 * *$ & $0.985(0.780-1.244)$ & 0.901 \\
\hline 列 & 0.070 & 0 & 0.00 & 0.01 \\
\hline USP21 & $0.903(0.807-1.011)$ & $0.077^{*}$ & $0.928(0.697-1.235)$ & 0.609 \\
\hline 1 & 0.836 & . & 0.884 & 0. \\
\hline ELTD1 & $1.155(1.021-1.307)$ & $0.021 * *$ & $0.969(0.772-1.216)$ & 0.787 \\
\hline 7 & 9) & * & 1.0 & 0.797 \\
\hline LASS6 & $0.867(0.782-0.961)$ & $0.006^{* *}$ & $0.788(0.631-0.984)$ & $0.035^{* *}$ \\
\hline SUOX2 & $1.076(0.975-1.187)$ & $0.141^{\prime}$ & $1.039(0.844-1.280)$ & 0.715 \\
\hline Age $(>60)$ & $0.154(0.01953-1.225)$ & $0.045^{* *}$ & $0.562(0.0354-2.392)$ & $0.049 * *$ \\
\hline Gender & $54-1.981)$ & 0.886 & $4-4.245)$ & 0.231 \\
\hline Stage & $1.644(0.8567-3.156)$ & $0.135^{\prime}$ & $1.274(0.494-3.286)$ & 0.615 \\
\hline
\end{tabular}

This table shows the univariate and multivariate cox proportional hazard regression analyses of 19 gene signatures and other clinical variables associated with overall survival of CRC patients. [Relevant location: Page 14]

\section{Discussion}

Microarray profiling allows the analysis of thousands of genes and the identification of differentially expressed genes which could then be used to characterize colorectal cancer from a molecular perspective. We performed a microarray study using the DASL assay on CRC patients with Dukes' B and C to predict patient's survival. This assay was designed with multiple probes per transcript to generate reproducible gene expression profiles from partially degraded RNA in archival tissues which had the advantage of information on the patients' survival. The quality of microarray data for downstream analysis is important in order to answer correctly the research questions. Outlier detection in microarray data is desirable to avoid noise and statistical damage with the aim to minimize the risk of misinterpreting the biological events.

This study has successfully stratified colorectal cancer using a 19-gene signature and provides a molecular staging approach of patients into low risk and high risk groups. The final aim is to use this identifier in a personalized approach for CRC patients as there are weaknesses in using histopathological examination alone to prognosticate patients' survival. The overall survival rate for CRC patients has increased, however, the individual survival rate for patients with Dukes' $B$ and $C$ is still low. A previous study developed a molecular classifier based on a core set of 43 genes to predict the 3-year survival for patients [12]. The gene signatures were also validated on a different population using a different platform. In this study, we identified gene signatures which could predict the 5-year survival rate. This is important to allow the best plan of treatment to be given to patients while at the same time reducing unnecessary toxicity and aggressive side effects.

The Oncotype Dx and Coloprint for colorectal cancers are two different assays developed based on quantitative multi-gene RT PCR assay and oligonucleotide microarray respectively. Both assays were developed to improve risk stratification of relapse for patients with Stage II CRC. Oncotype Dx has a limitation in which identified genes were derived from four separate studies on individual genes and not determined using the whole genome microarray method. Therefore, the assay could probably miss important genes that may be involved in determining cancer relapse. Another disadvantage of the assay is the difficulty in assigning patients into groups of risk prediction due to the narrow range of prediction scores. These challenges hinder the effective use of this assay in clinical practice [25]. The gene expression data from Coloprint was not made publicly available hence the evaluation of the classifier cannot be performed [14]. A recent study developed 113 gene signatures from a published gene expression profile to predict prognostic risk [26]. Prognostic index for patients was calculated to discriminate patients into high- and low risk group. To show the prognostic significance, validation was done using independent data sets from different countries using the same platform. In this study, we completed the whole genome microarray and calculated a risk score for each patient. We used the median risk score as a cutoff point in which the median was not affected by outliers [27]. Using this median, the patients were efficiently separated into two groups i.e., high and low risk groups.

Previous clinical trials have used two robust gene classifiers called ColoGuide Ex and ColoGuide Pro. The investigators used the classifiers to stratify the prognosis of patients with stage II and III [14, 15]. The performances of both classifiers were validated using independent external datasets from different countries. The strength of the classifier is that it requires validation at the individual patient level conducted in the prospective trial. In our study, we revealed a set of genes which could provide a significant risk assessment approach for patients in both intermediate stages (Dukes' B and C). Therefore, an 
Table 4 Comparison the LASSO and Ridge regression methods with Elastic Net regression

\begin{tabular}{|c|c|c|c|c|c|}
\hline & & \multicolumn{2}{|l|}{ Univariate } & \multicolumn{2}{|l|}{ Multivariate } \\
\hline & & $\mathrm{HR}(95 \% \mathrm{Cl})$ & $P$ & $\mathrm{HR}(95 \% \mathrm{Cl})$ & $P$ \\
\hline \multirow[t]{2}{*}{ Datasets } & Methods & & & & \\
\hline & Lasso & $0.106(0.030-0.370)$ & 0.000 & $0.063(0.016-0.252)$ & 0.000 \\
\hline \multirow[t]{2}{*}{ Our dataset } & Ridge & $0.812(0.303-2.173)$ & 0.000 & $0.083(0.022-0.321)$ & 0.000 \\
\hline & Elastic net & $0.065(0.014-0.287)$ & 0.000 & $0.040(0.008-0.198)$ & 0.000 \\
\hline \multirow[t]{3}{*}{ Denmark dataset } & Lasso & $0.055(0.007-0.453)$ & 0.007 & $0.044(0.005-0.396)$ & 0.005 \\
\hline & Ridge & $0.112(0.024-0.519)$ & 0.005 & $0.080(0.014-0.467)$ & 0.005 \\
\hline & Elastic net & $0.057(0.007-0.464)$ & 0.007 & $0.038(0.004-0.389)$ & 0.005 \\
\hline \multirow[t]{3}{*}{ Australian dataset } & Lasso & $0.565(0.396-0.805)$ & 0.002 & $0.549(0.384-0.784)$ & 0.001 \\
\hline & Ridge & $0.447(0.312-0.641)$ & 0.000 & $0.454(0.316-0.651)$ & 0.000 \\
\hline & Elastic net & $0.523(0.370-0.739)$ & 0.000 & $0.529(0.373-0.748)$ & 0.000 \\
\hline \multirow[t]{3}{*}{ USA dataset } & Lasso & $0.105(0.010-1.068)$ & 0.056 & $0.104(0.010-1.052)$ & 0.055 \\
\hline & Ridge & $0.130(0.013-1.294)$ & 0.082 & $0.129(0.013-1.283)$ & 0.081 \\
\hline & Elastic net & $0.120(0.012-1.195)$ & 0.071 & $0.122(0.012-1.214)$ & 0.072 \\
\hline \multirow[t]{3}{*}{ Norway dataset } & Lasso & - & - & - & - \\
\hline & Ridge & - & - & - & - \\
\hline & Elastic net & $0.536(0.300-0.957)$ & 0.035 & $0.569(0.318-1.018)$ & 0.057 \\
\hline
\end{tabular}

This table shows the comparison with the LASSO, Ridge regression and Elastic Net methods for 19 gene signatures based on our dataset and other external datasets from different countries. Univariate and multivariate Cox's proportional hazard model analysis of prognostic factor (prognostic index or risk score) for overall survival [Relevant location: Page 16]

Table 5 The probe ID, gene symbols and expression of the 19 genes in the poor survival group compared to the good survival group

\begin{tabular}{|c|c|c|c|}
\hline Probe ID & Gene symbol & Gene name & $\begin{array}{l}\text { Expression in poor survival group } \\
\text { (Up-regulated/Down-regulated) }\end{array}$ \\
\hline 7000692 & MRPL52 & mitochondrial ribosomal protein $L 52$ & Down-regulated \\
\hline 5700373 & TRIP13 & thyroid hormone receptor interactor 13 & Down-regulated \\
\hline 2690324 & ITPRIP & inositol 1,4,5-triphosphate receptor interacting protein & Up-regulated \\
\hline 7000184 & SLC38A9 & solute carrier family 38, member 9 & Down-regulated \\
\hline 5420070 & FRMD6 & FERM domain containing 6 & Up-regulated \\
\hline 4230739 & SORCS2 & sortilin-related VPS10 domain containing receptor 2 & Up-regulated \\
\hline 6040070 & ELTDI & EGF, latrophilin and seven transmembrane domain containing 1 & Up-regulated \\
\hline 1190176 & NOTCH2 & Notch homolog 2 & Up-regulated \\
\hline 2570196 & CPXCR1 & CPX chromosome region, candidate 1 & Down-regulated \\
\hline 840367 & $\mathrm{OR} 10 \mathrm{H} 5$ & olfactory receptor, family 10 , subfamily $\mathrm{H}$, member 5 & Down-regulated \\
\hline 3450575 & $P D C$ & phosducin & Down-regulated \\
\hline 2710564 & DUOX2 & dual oxidase 2 & Up-regulated \\
\hline 4560474 & GFRA4 & GDNF family receptor alpha 4 & Down-regulated \\
\hline 5690064 & LASS6 & LAG1 homolog, ceramide synthase 6 & Down-regulated \\
\hline 3780725 & OSBPL9 & oxysterol binding protein-like 9 & Down-regulated \\
\hline 5090025 & C12orf66 & chromosome 12 open reading frame 66 & Down-regulated \\
\hline 5870121 & SPG7 & spastic paraplegia 7 (pure and complicated autosomal recessive) & Up-regulated \\
\hline 620398 & DUSP21 & dual specificity phosphatase 21 & Down-regulated \\
\hline 540411 & $B R C A 1$ & breast cancer 1 , early onset & Down-regulated \\
\hline
\end{tabular}


qPCR

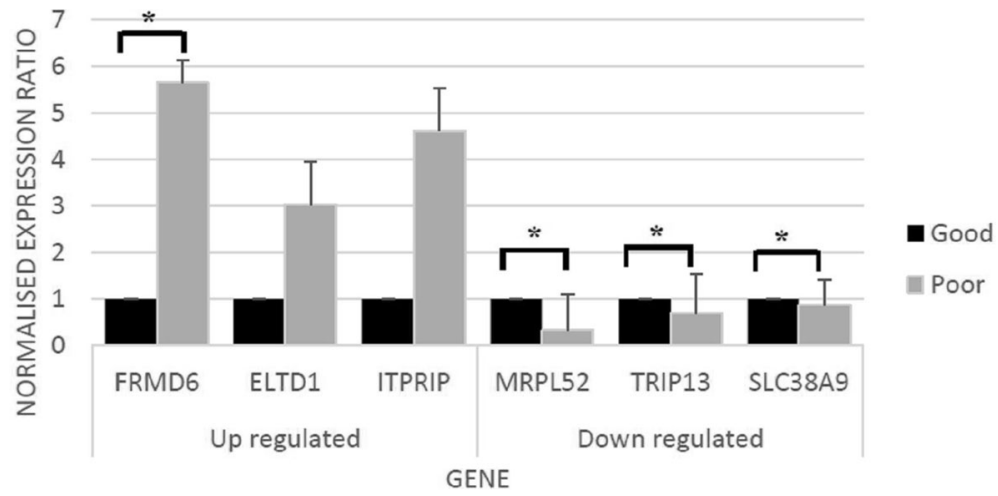

Fig. 4 Validation of detected genes using qPCR. The normalized gene expression ratio for six genes including FRMD6, ELTD1, ITPRIP, MRPL52, TRIP13 and SLC38A9 which was determined using qPCR $(p<0.05)$. $\left(^{*}\right)$ represents the significant genes

inadequate sampling of lymph nodes as a risk factor in the conventional clinico-pathologic approach to determine staging could be disregarded [16, 28].

From our findings, some of the signature genes play roles in cell differentiation and amino acid transport as well as coding for phosphoproteins, receptors and membrane-associated proteins. We validated and confirmed six out of the 19 genes using RT-PCR. One of these is the EGF latrophilin and seven transmembrane domain-containing protein 1 (ELTD1) gene. A previous in vivo study found that a high expression ( 3 fold increase, $p<0.001)$ of this gene was associated with high grade gliomas and low survival rate [29]. In our study, ELTDI was consistently up-regulated in the group of CRC patients with poor survival. The ELTDI gene is a member of the secretin family of $G$ protein-coupled peptide hormone receptors and belongs to the EGF-7 transmembrane subfamily. The EGF family plays important roles in cell division, apoptosis, differentiation and migration [30]. Wallgard et al. [31] reported that ELDT1 is associated with microvasculature expression of endothelial cell-specific in vivo for tumor progression.

The second validated gene, thyroid hormone receptor interactor 13 (TRIP13), encodes a protein that cooperates with thyroid hormone receptors. High expression of TRIP13 gene was reported to be associated with poor prognosis in breast cancer [32]. One of the gene in this panel was the sortilin-related VPS10 domain containing receptor 2 (SORCS2) which found to be up-regulated in the group with poor survival compared to those with good survival. This gene is normally highly expressed in the central nervous system during development [33, 34]. In contrast, this gene was documented to be down-regulated in the stromal cells of breast cancer and associated with poor outcome [35, 36]. Another one of the genes in the panel i.e., Notch homolog 2 (NOTCH2) has been widely reported to be linked with survival. $\mathrm{NOTCH} 2$ is a receptor for membrane bound ligands and has roles in vascular, renal and hepatic development $[37,38]$. The NOTCH2 gene was also reported to be an independent prognostic predictor of $\mathrm{CRC}$ [39]. A high expression of $\mathrm{NOTCH} 2$ might predict good survival in CRC with a median survival of 45 months [39]. We noted an opposite effect of this gene in our current study. This is probably due to the heterogeneity of tissue samples with different stages of tumor tissues between the respective studies. Less information are available for six of the genes in the panel in relation to cancer namely the ITPRIP, FRMD6, CPXCR1, SLC38A9, MRPL52 and GFRA4.

We showed that the performance of the 19-gene signature was reliable and also reproducible. This was evident through the use of four external validation series from other countries with different population settings. A similar study used information from other studies to validate the performance of their 5-gene panel [40]. Our study results show the robustness of the gene panel whereby the test successfully differentiate patients into groups with high and low risk of recurrence in the training, testing and also the external validation datasets.

\section{Conclusions}

We have shown that our 19-gene signature is able to classify CRC patients into prognostic groups according to their risk scores. Patient who will be assigned into the high risk group could have a proper treatment plan, relevant chemotherapy dosage and effective medication strategy in order to increase the survival rate at the same time reduce the invasiveness of cancer cells. While for patients who were classified as the low risk group could avoid or received lower doses of adjuvant chemotherapy. Further validation tests are still required using larger number of samples. Future prospective clinical trials could also be conducted using this classifier to randomize treatment groups and explore further the sensitivity and specificity of this 19-gene signature. 


\section{Additional file}

Additional file 1: a) Detection of outliers. b) Summary of permutation analysis. a) Detection of outliers. Histogram of microarray data showing the presence of outliers after detection by using the beta weight function. Samples with weight index $<0.2$ were considered as outliers. b) Summary of the permutation analysis for 78 CRC samples to identify differentially expressed genes. One hundred training and test set were generated and further analyzed using 3 statistical methods: SAM, LIMMA and $t$-test to calculate the $\mathrm{p}$-value for each gene. (DOCX $452 \mathrm{~kb}$ )

\section{Acknowledgment}

The authors wish to thank National Registration Department of Malaysia for their assistance throughout the project.

\section{Funding}

The project was funded by the Higher Institution Centre of Excellence (HiCOE) grant (JJ-003-2011) from the Ministry of Higher Education. The funders had no role in study design, data collection and analysis, decision to publish, or preparation of the manuscript.

\section{Availability of data and material}

The datasets generated during and/or analyzed during the current study are not publicly available due to the huge datasets but are available from the corresponding author on reasonable request.

\section{Authors' contributions}

Conceived and designed the experiments: NMM RJ RH IMR IS. Performed the experiments: NAA. Analyzed the data: NAA MHM NMM. Contributed reagents/materials/analysis tools: RJ, NMM. Wrote the paper: NAA NMM RJ. All authors read and approved the final manuscript.

\section{Competing interests}

The authors declare that they have no competing interests.

\section{Consent for publication}

Not applicable.

\section{Ethical approval and consents to participate}

The study was approved by the institutional ethics committee (Ref. No.: UKM 1.5.3.5/244/SPP2).

\section{Author details \\ 'UKM Medical Molecular Biology Institute, Universiti Kebangsaan Malaysia (UKM), Cheras, Kuala Lumpur, Malaysia. '2Department of Physiology, Faculty of Medicine, Universiti Kebangsaan Malaysia, Jalan Yaacob Latif, Bandar Tun Razak, Cheras, 56000 Kuala Lumpur, Malaysia. ${ }^{3}$ Histopathology Unit, Department of Pathology, Universiti Kebangsaan Malaysia Medical Centre Kuala Lumpur, Malaysia. ${ }^{4}$ Department of Surgery, Universiti Kebangsaan Malaysia Medical Centre, Kuala Lumpur, Malaysia. ${ }^{5}$ Department of Community Health, Faculty of Medicine, Universiti Kebangsaan Malaysia, Kuala Lumpur, Malaysia.}

Received: 23 September 2015 Accepted: 24 August 2016 Published online: 08 September 2016

\section{References}

1. Ferlay J, Soerjomataram I, Dikshit R, Eser S, Mathers C, Rebelo M, Parkin DM, Forman D, Bray F. Cancer incidence and mortality worldwide-sources, methods and major patterns in GLOBOCAN 2012. Int J Cancer. 2015;136(5):E359-86. doi:10.1002/ijc.29210

2. Wu JS. Rectal cancer staging. Clin Colon Rectal Surg. 2007;20(3):148-57. doi:10.1055/s-2007-984859.

3. Morris EJ, Maughan NJ, Forman D, Quirke P. Who to treat with adjuvant therapy in Dukes B/stage II colorectal cancer? The need for high quality pathology. Gut. 2007;56(10):1419-25. doi:10.1136/gut.2006.116830.

4. O'Connell JB, Maggard MA, Ko CY. Colon cancer survival rates with the new American Joint Committee on Cancer sixth edition staging. J Natl Cancer Inst. 2004;96(19):1420-5. doi:10.1093/jnci/djh275.
5. Ahn JB, Chung HC, Yoo NC, Roh JK, Kim NK, Suh CO, Kim GE, Seong JS, Shim WH. Efficacy of postoperative concurrent chemoradiation for resectable rectal cancer: a single institute experience. Cancer Res Treat. 2004:36(4):228-34. doi:10.4143/crt.2004.36.4.228.

6. Marisa L, de Reynies A, Duval A, Selves J, Gaub MP, Vescovo L, EtienneGrimaldi MC, Schiappa R, Guenot D, Ayadi M, et al. Gene expression classification of colon cancer into molecular subtypes-characterization, validation, and prognostic value. PLoS Med. 2013;10(5):e1001453. doi:10.1371/journal.pmed.1001453.

7. Marshall JL, Haller DG, de Gramont A, Hochster HS, Lenz HJ, Ajani JA, Goldberg RM. Adjuvant therapy for stage II and III colon cancer: Consensus Report of the International Society of Gastrointestinal Oncology. Gastrointest Cancer Res. 2007:1(4):146-54.

8. Irizarry RA, Bolstad BM, Collin F, Cope LM, Hobbs B, et al. Summaries of Affymetrix GeneChip probe level data. Nucleic Acids Res. 2003;31:e15.

9. Wu Y, Wang X, Wu F, Huang R, Xue F, Liang G, Tao M, Cai P, Huang Y. Transcriptome profiling of the cancer, adjacent non-tumor and distant normal tissues from a colorectal cancer patient by deep sequencing. PLoS One. 2012;7(8):e41001. doi:10.1371/journal.pone.0041001.

10. Wang $Y$, Jatkoe $T$, Zhang $Y$, Mutch MG, Talantov D, Jiang J, McLeod HL, Atkins D. Gene expression profiles and molecular markers to predict recurrence of Dukes' B colon cancer. J Clin Oncol. 2004;22(9):1564-71. doi:10.1200/JCO.2004.08.186

11. Jorissen RN, Gibbs P, Christie M, Prakash S, Lipton L, Desai J, Kerr D, Aaltonen LA, Arango D, Kruhoffer M, et al. Metastasis-Associated gene expression changes predict poor outcomes in patients with Dukes Stage B and C colorectal cancer. Clin Cancer Res. 2009;15(24):7642-51. doi:10.1158/1078-0432.CCR-09-1431.

12. Eschrich S, Yang I, Bloom G, Kwong KY, Boulware D, Cantor A, Coppola D, Kruhoffer M, Aaltonen L, Orntoft TF, et al. Molecular staging for survival prediction of colorectal cancer patients. J Clin Oncol. 2005;23(15):3526-35. doi:10.1200/JCO.2005.00.695.

13. Jiang WQ, Fu FF, Li YX, Wang WB, Wang HH, Jiang HP, Teng LS. Molecular biomarkers of colorectal cancer: prognostic and predictive tools for clinical practice. J Zhejiang Univ Sci B. 2012;13(9):663-75. doi:10.1631/jzus.B1100340.

14. Agesen TH, Sveen A, Merok MA, Lind GE, Nesbakken A, Skotheim RI, Lothe RA. ColoGuideEx: a robust gene classifier specific for stage II colorectal cancer prognosis. Gut. 2012;61(11):1560-7. doi:10.1136/gutjnl-2011-301179.

15. Sveen A, Agesen TH, Nesbakken A, Meling Gl, Rognum TO, Liestol K, Skotheim RI, Lothe RA. ColoGuidePro: a prognostic 7-gene expression signature for stage III colorectal cancer patients. Clin Cancer Res. 2012;18(21):6001-10. doi:10.1158/1078-0432.CCR-11-3302.

16. Salazar R, Roepman P, Capella G, Moreno V, Simon I, Dreezen C, LopezDoriga A, Santos C, Marijnen C, Westerga J, et al. Gene expression signature to improve prognosis prediction of stage II and III colorectal cancer. J Clin Oncol. 2011;29(1):17-24. doi:10.1200/JCO.2010.30.1077.

17. Fung KY, Nice E, Priebe I, Belobrajdic D, Phatak A, Purins L, Tabor B, Pompeia C, Lockett T, Adams TE, et al. Colorectal cancer biomarkers: to be or not to be? Cautionary tales from a road well travelled. World J Gastroenterol. 2014;20(4):888-98. doi:10.3748/wjg.v20.i4.888.

18. Lu AT, Salpeter SR, Reeve AE, Eschrich S, Johnston PG, Barrier AJ, Bertucci F, Buckley NS, Salpeter EE, Lin AY. Gene expression profiles as predictors of poor outcomes in stage II colorectal cancer: A systematic review and meta-analysis. Clin Colorectal Cancer. 2009:8(4):207-14. doi:10.3816/CCC.2009.n.035

19. Mollah MM, Mollah MN, Kishino H. Beta-empirical Bayes inference and model diagnosis of microarray data. BMC Bioinformatics. 2012;13:135. doi:10.1186/1471-2105-13-135.

20. Gottardo R, Raftery AE, Yeung KY, Bumgarner RE. Bayesian robust inference for differential gene expression in microarrays with multiple samples. Biometrics. 2006;62(1):10-8. doi:10.1111/j.1541-0420.2005.00397.x.

21. Mollah MN, Sultana N, Minami M, Eguchi S. Robust extraction of local structures by the minimum beta-divergence method. Neural Netw. 2010;23(2):226-38. doi:10.1016/j.neunet.2009.11.011.

22. Mollah MN, Eguchi S. Robust QTL analysis by minimum beta-divergence method. Int J Data Min Bioinform. 2010;4(4):471-85.

23. Waldron L, Pintilie M, Tsao MS, Shepherd FA, Huttenhower C, Jurisica I. Optimized application of penalized regression methods to diverse genomic data. Bioinformatics. 2011:27(24):3399-406. doi:10.1093/ bioinformatics/btr591.

24. Thorsteinsson M, Kirkeby LT, Hansen R, Lund LR, Sorensen LT, Gerds TA, Jess P, Olsen J. Gene expression profiles in stages II and III colon 
cancers: application of a 128-gene signature. Int J Colorectal Dis. 2012;27(12):1579-86. doi:10.1007/s00384-012-1517-4.

25. Webber EM, Lin JS, Evelyn PW. Oncotype DX tumor gene expression profiling in stage II colon cancer. Application: prognostic, risk prediction. PLoS Curr. 2010;2. doi: 10.1371/Currents.RRN1177

26. Nguyen MN, Choi TG, Nguyen DT, Kim JH, Jo YH, Shahid M, Akter S, Aryal SN Yoo JY, Ahn YJ, et al. RC-113 gene expression signature for predicting prognosis in patients with colorectal cancer. Oncotarget. 2015;6(31):31674-92. doi:10.18632/oncotarget.5183.

27. Larson MG. Descriptive statistics and graphical displays. Circulation. 2006;1 14(1):76-81. doi:10.1161/CIRCULATIONAHA.105.584474.

28. Le Voyer TE, Sigurdson ER, Hanlon AL, Mayer RJ, Macdonald JS, Catalano PJ, Haller DG. Colon cancer survival is associated with increasing number of lymph nodes analyzed: a secondary survey of intergroup trial INT-0089. J Clin Oncol. 2003:21(15):2912-9. doi:10.1200/JCO.2003.05.062.

29. Towner RA, Jensen RL, Colman H, Vaillant B, Smith N, Casteel R, Saunders D, Gillespie DL, Silasi-Mansat R, Lupu F, et al. ELTD1, a potential new biomarker for gliomas. Neurosurgery. 2013;72(1):77-90. doi:10.1227/NEU. 0b013e318276b29d. discussion 91.

30. Normanno N, De Luca A, Bianco C, Strizzi L, Mancino M, Maiello MR, Carotenuto A, De Feo G, Caponigro F, Salomon DS. Epidermal growth factor receptor (EGFR) signaling in cancer. Gene. 2006;366(1):2-16. doi:10.1016/j. gene.2005.10.018.

31. Wallgard E, Larsson E, He L, Hellstrom M, Armulik A, Nisancioglu MH, Genove G, Lindahl P, Betsholtz C. Identification of a core set of 58 gene transcripts with broad and specific expression in the microvasculature. Arterioscler Thromb Vasc Biol. 2008;28(8):1469-76. doi:10.1161/ATVBAHA. 108.165738.

32. Martin KJ, Patrick DR, Bissell MJ, Fournier MV. Prognostic breast cancer signature identified from 3D culture model accurately predicts clinical outcome across independent datasets. PLoS One. 2008;3(8):e2994. doi:10.1371/journal.pone.0002994.

33. Hermey G, Plath N, Hubner CA, Kuhl D, Schaller HC, Hermans-Borgmeyer I. The three sorCS genes are differentially expressed and regulated by synaptic activity. J Neurochem. 2004;88(6):1470-6.

34. Rezgaoui M, Hermey G, Riedel IB, Hampe W, Schaller HC, HermansBorgmeyer I. Identification of SorCS2, a novel member of the VPS10 domain containing receptor family, prominently expressed in the developing mouse brain. Mech Dev. 2001;100(2):335-8.

35. Wu X, Ye Y, Rosell R, Amos Cl, Stewart DJ, Hildebrandt MA, Roth JA, Minna JD, Gu J, Lin J, et al. Genome-wide association study of survival in non-small cell lung cancer patients receiving platinum-based chemotherapy. J Natl Cancer Inst. 201 1;103(10):817-25. doi:10.1093/jnci/djr075.

36. Finak $G$, Bertos $N$, Pepin F, Sadekova S, Souleimanova M, Zhao H, Chen H, Omeroglu G, Meterissian S, Omeroglu A, et al. Stromal gene expression predicts clinical outcome in breast cancer. Nat Med. 2008;14(5):518-27. doi:10.1038/nm1764.

37. McCright B. Notch signaling in kidney development. Curr Opin Nephrol Hypertens. 2003;12(1):5-10. doi:10.1097/01.mnh.0000049802.69874.c0.

38. Lemaigre F, Zaret KS. Liver development update: new embryo models, cell lineage control, and morphogenesis. Curr Opin Genet Dev. 2004;14(5):582-90. doi:10.1016/.j.gde.2004.08.004

39. Chu D, Zhang Z, Zhou Y, Wang W, Li Y, Zhang H, Dong G, Zhao Q, Ji G. Notch1 and Notch2 have opposite prognostic effects on patients with colorectal cancer. Ann Oncol. 2011;22(11):2440-7. doi:10.1093/annonc/mda776.

40. Lenehan PF, Boardman LA, Riegert-Johnson D, De Petris G, Fry DW, Ohrnberger J, Heyman ER, Gerard B, Almal AA, Worzel WP. Generation and external validation of a tumor-derived 5-gene prognostic signature for recurrence of lymph node-negative, invasive colorectal carcinoma. Cancer. 2012;118(21):5234-524. doi:10.1002/cncr.27628.

\section{Submit your next manuscript to BioMed Central and we will help you at every step:}

- We accept pre-submission inquiries

- Our selector tool helps you to find the most relevant journal

- We provide round the clock customer support

- Convenient online submission

- Thorough peer review

- Inclusion in PubMed and all major indexing services

- Maximum visibility for your research

Submit your manuscript at www.biomedcentral.com/submit
C Biomed Central 